\title{
cansm \\ Carbon Nanotube Far Infrared Detectors with High Responsivity and Superior Polarization Selectivity Based on Engineered Optical Antennas
}

\author{
Xiansong Ren, Zhaoyu Ji, Binkai Chen, Jing Zhou *, Zeshi Chu and Xiaoshuang Chen * \\ State Key Laboratory of Infrared Physics, Shanghai Institute of Technical Physics, Chinese Academy of Sciences, \\ Shanghai 200083, China; renxs@bupt.edu.cn (X.R.); jizhaoyu@outlook.com (Z.J.); 13120666402@163.com (B.C.); \\ chuzeshi@mail.sitp.ac.cn (Z.C.) \\ * Correspondence: jzhou@mail.sipt.ac.cn (J.Z.); xschen@mail.sitp.ac.cn (X.C.); Tel.: +86-21-2505-1889 (J.Z.); \\ +86-21-2505-1403 (X.C.)
}

Citation: Ren, X.; Ji, Z.; Chen, B.; Zhou, J.; Chu, Z.; Chen, X. Carbon Nanotube Far Infrared Detectors with High Responsivity and Superior Polarization Selectivity Based on Engineered Optical Antennas. Sensors 2021, 21, 5221. https://doi.org/ $10.3390 / \mathrm{s} 21155221$

Academic Editor: Dragan Indjin

Received: 16 July 2021

Accepted: 27 July 2021

Published: 31 July 2021

Publisher's Note: MDPI stays neutral with regard to jurisdictional claims in published maps and institutional affiliations.

Copyright: (c) 2021 by the authors. Licensee MDPI, Basel, Switzerland. This article is an open access article distributed under the terms and conditions of the Creative Commons Attribution (CC BY) license (https:/ / creativecommons.org/licenses/by/ $4.0 /)$.

\begin{abstract}
Single-wall carbon nanotube (SWCNT) thin films are promising for sensitive uncooled infrared detection based on the photothermoelectric effect. The SWCNT film is usually shaped into a belt and diversely doped to form a p-n junction at the center. Under the illumination of a focused incident light, the temperature gradient from the junction to the contacts leads to photoresponse. When the SWCNTs are aligned in one direction, the photoresponse becomes polarization selective. Although a typical bowtie antenna can improve the responsivity and polarization extinction ratio by deep-subwavelength light focusing, the absolute absorptance of the junction region is only $0.6 \%$. In this work, the antenna was engineered for a higher light coupling efficiency. By integrating a bottom metal plane at a specific distance from the SWCNT film and optimizing the antenna geometries, we achieved ultra-efficient impedance matching between the antenna and the SWCNTs, thus the absorptance of the junction region was further enhanced by 21.3 times and reached $13.5 \%$, which is more than 3 orders of magnitude higher than that of the device without the engineered antenna. The peak responsivity was further enhanced by 19.9 times and responsivity reached $1500 \mathrm{~V} / \mathrm{W}$ at $1 \mathrm{THz}$. The resonant frequency can be tuned by changing the size of the antenna. Over the frequency range of $0.5 \mathrm{THz}$ to $1.5 \mathrm{THz}$, the peak responsivity was further enhanced by 8.1 to 19.9 times, and the polarization extinction ratio was enhanced by 2.7 to 22.3 times. The highest polarization extinction ratio reached $3.04 \times 10^{5}$ at $0.5 \mathrm{THz}$. The results are based on the numerical simulations of the light and the thermal fields.
\end{abstract}

Keywords: carbon nanotube far infrared detectors; optical antenna engineering; responsivity enhancement; polarization sensitivity enhancement; analysis of coupled light and thermal fields

\section{Introduction}

Far infrared (including $\mathrm{THz}$ ) detectors are important to a wide range of applications in the fields of astronomy, sensing, spectroscopy, imaging, defense and communications [1-3]. Since the photon energy in this regime is quite low, photonic detectors require cryogenic cooling systems, which are expensive and bulky, to maintain the performance. In this respect, thermal detectors have an advantage as they can operate in an uncooled mode, although they are generally less sensitive and slower than photonic detectors [4]. In this situation, new materials with a prominent thermoelectric effect, low specific heat capacity, and high thermal stability become promising candidates for future infrared thermal detectors [5-12]. Among those materials, single-wall carbon nanotubes (SWCNT) have attracted great attention due to their unique electrical, optical and thermal properties [4,8-14]. In particular, SWCNT thin films have been proposed and demonstrated as a potential infrared detection material by a lot of researchers, since they can be made highly uniform and can be diversely doped to form $p-n$ junctions $[8,13,14]$. In addition, they are compatible 
with a variety of substrates. For a SWCNT thin film with a p-n junction, the far infrared illumination at the junction area will induce a self-driven photoresponse proportional to the difference between the Seebeck coefficients of the $\mathrm{p}$ - and n-doped parts and also proportional to the temperature rise at the junction, according to the PTE effect. When the assembled SWCNTs are aligned perpendicular to the electronic transportation direction, the reduced thermal conductivity benefits the local temperature rise at the junction area and then enhances the responsivity $[9,13,14]$. As a positive side effect, the alignment of the SWCNTs with intrinsic anisotropy leads to polarization selectivity, which is essential for infrared polarimetry.

Optical antennas have been widely employed to focus electromagnetic radiation into an area much smaller than the diffraction limit [15-17] and have been demonstrated to be able to enhance the responsivity of the aligned SWCNT film infrared detectors by 1 to 2 orders of magnitude and the polarization extinction ratio by 2 order of magnitudes [18]. Although it greatly improves the performance of SWCNTs-type infrared detectors, the absolute absorptance of the junction region (a $100 \mu \mathrm{m} \times 5 \mu \mathrm{m} \times 2 \mu \mathrm{m}$ region at the $\mathrm{p}-\mathrm{n}$ junction) is only $0.6 \%$. In this work, we propose to significantly enhance the absorptance of the junction region (deep-subwavelength) in an aligned SWCNT film by engineering the optical antenna. A metal plane is added to induce a mirror image of the induced charges in the antenna. By setting the metal plane at a specific distance from the antenna, the radiation from the mirror image can interfere with that from the antenna in a proper manner, leading to a controlled radiation quality factor $\left(Q_{\mathrm{e}}\right)$ that matches the absorption quality factor $\left(Q_{\mathrm{r}}\right)$ for an optimized coupling efficiency. In another parameter dimension, the extension bar at each feeding point tips of the antenna is engineered to match the load resistance $\left(R_{\text {load }}\right)$ with the radiation resistance $\left(R_{\mathrm{rad}}\right)$. When $R_{\text {load }}$ equals to $R_{\mathrm{rad}}$, the antenna exhibits a maximum coupling efficiency. By engineering the light coupling properties of the antenna, the absorptance of the junction region is further increased by 21.3 times and reaches $13.5 \%$. This absorptance is more than 3 orders of magnitude higher than that in the absence of the engineered antenna. Based on the coupled simulation of the light field and the thermal field, the temperature rise at the junction is further enhanced by 19.9 times compared to the case of an ordinary antenna integrated SWCNT film, and the responsivity $\left(R_{\mathrm{V}}\right)$ reaches $1500 \mathrm{~V} / \mathrm{W}$ at $1 \mathrm{THz}$. $R_{\mathrm{V}}$ is defined as the photovoltage divided by the incident optical power on the $100 \mu \mathrm{m} \times 5 \mu \mathrm{m}$ area at the junction. The resonant frequency can be tuned by changing the size of the antenna. Over the range of 0.5 to $1.5 \mathrm{THz}$, the engineered antenna enhances the peak responsivities further by 8.1 to 19.9 times and enhances the peak polarization extinction ratios by 2.7 to 22.3 times, compared with the ordinary bowtie antenna [18]. It is worth noting that the peak polarization extinction ratio is higher than 1481 over the range from 0.5 to $1.5 \mathrm{THz}$ and reaches an extremely high value of $3.04 \times 10^{5}$ at $0.5 \mathrm{THz}$.

\section{Materials and Methods}

\subsection{Aligned SWCNT Thin Film as an Effective Uniaxial Medium}

A typical aligned SWCNT thin film infrared detector is shown in Figure 1a. The SWCNT film is considered as an effective uniaxial medium $[18,19]$ because the diameter of each SWCNT and the inter-distances between them are within the deep subwavelength scale. The effective permittivity tensor is a diagonal matrix, as shown below $[18,19]$ :

$$
\begin{gathered}
\varepsilon_{\mathrm{x}}=\varepsilon_{\mathrm{z}}=\varepsilon_{\perp}=\varepsilon_{\mathrm{e}}+f \varepsilon_{\mathrm{e}} \frac{\varepsilon_{\text {SWCNT }}-\varepsilon_{\mathrm{e}}}{\varepsilon_{\mathrm{e}}+\frac{(1-f)\left(\varepsilon_{\text {SWCNT }}-\varepsilon_{\mathrm{e}}\right)}{2}} \\
\varepsilon_{\mathrm{y}}=\varepsilon_{\|}=\varepsilon_{\mathrm{e}}+f \varepsilon_{\mathrm{e}}\left(\varepsilon_{\text {SWCNT }}-\varepsilon_{\mathrm{e}}\right)
\end{gathered}
$$

$\varepsilon_{\perp}$ denote the relative permittivity of an aligned SWCNT film for the light field polarized perpendicular to the SWCNTs, and $\varepsilon_{\|}$denote the relative permittivity for the light field parallel to the SWCNTs. $f$ represents the fill factor, $\varepsilon_{S W C N T}$ is the permittivity of a single SWCNT, which can be approximated as a solid $\operatorname{rod}[20,21]$, and $\varepsilon_{\mathrm{e}}$ is the permittivity of the 
environment, which is air in this case. Based on the density of a SWCNT film [22,23], we assume $f=0.3$. Since the SWCNT film contains both semiconductor nanotubes and metal nanotubes, $\varepsilon_{\mathrm{CNT}}$ represents the average optical response. Based on the actual measurement results of SWCNT film under far-infrared conditions [24,25], $\varepsilon_{\mathrm{CNT}}$ is obtained through effective medium theory and modeled in the Drude-Lorentz form [20]. Based on this, we obtained the permittivity $\varepsilon_{\perp}$ and $\varepsilon_{\|}$of SWCNT.

(a)

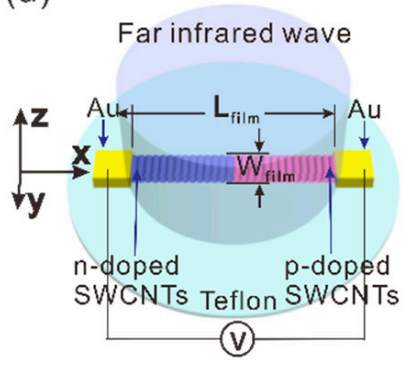

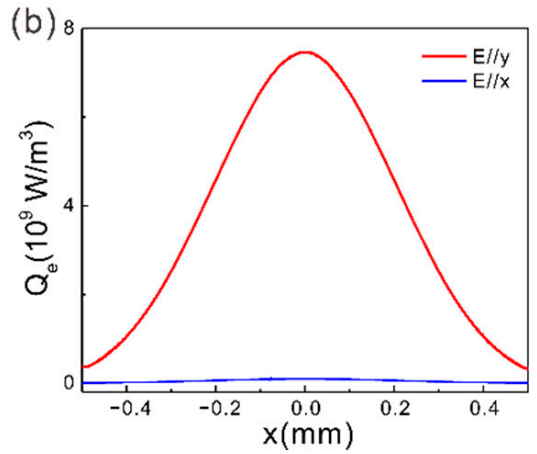

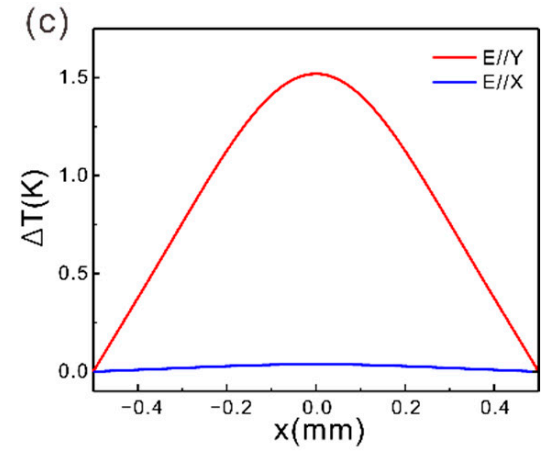

Figure 1. (a) Schematic of the aligned SWCNT film based far-infrared photodetector. $L_{\text {film }}=1 \mathrm{~mm}, W_{\text {film }}=5 \mu \mathrm{m}$. (b) Light power absorption density in the aligned SWCNT film $\left(Q_{\mathrm{e}}\right)$ versus $x$ at $y=0 \mu \mathrm{m}$ and $z=1 \mu \mathrm{m}$ for $y$-polarized incident light (Red line) and that for $x$-polarized incident light (Blue line). (c) $\Delta T$ versus $x$ at $y=0 \mu \mathrm{m}$ and $z=1 \mu \mathrm{m}$ for $y$-polarized incident light (Red line) and that for $x$-polarized incident light (Blue line).

\subsection{Responsivity Obtained by Optical and Thermal Field Simulation}

As shown in Figure 1a, the aligned SWCNT film $(1 \mathrm{~mm}$ long, $5 \mu \mathrm{m}$ wide, and $2 \mu \mathrm{m}$ thick) is supported by a transparent dielectric substrate (teflon or SU8), and connected by two metal contacts. By diversely doping the two halves of the SWCNT film, a p-n junction is formed at the center. The Seebeck coefficient is positive in the p-doped region and negative in the n-doped region. In this way, when the incident light is focused at the p-n junction, a distributed temperature rise is created. The temperature at the junction is the highest and gradually decreases from the center to the two ends. Then, a photoresponse is created due to the photothermoelectric effect. The SWCNT film can be formed by first growing vertically and rolling down to form a macroscopic film [13,14,22], and then patterning the film by photolithography and etching $[26,27]$. The photoresponse can be estimated by numerical simulations of the optical field and the thermal field. The light field and the thermal field are described by the following equations (Equations (1)-(3)):

$$
\begin{gathered}
\nabla^{2} \boldsymbol{E}-k_{0}^{2} \stackrel{\leftrightarrow}{\varepsilon}_{\mathrm{r}} \boldsymbol{E}=0, \\
-\nabla \cdot\left(\stackrel{\leftrightarrow}{k} \nabla^{2} T\right)=Q_{\mathrm{e}} \\
Q_{\mathrm{e}}=\frac{1}{2} \varepsilon_{0} \omega\left(\boldsymbol{E} \cdot\left(\operatorname{Im}\left(\stackrel{\leftrightarrow}{\varepsilon}_{\mathrm{r}}\right) \cdot \boldsymbol{E}\right)\right) .
\end{gathered}
$$

where $k_{0}$ represents the wave vector in vacuum, $\stackrel{\leftrightarrow}{\varepsilon}_{\mathrm{r}}$ is the relative permittivity tensor of the aligned SWCNT film, $\stackrel{\leftrightarrow}{k}$ is the thermal conductivity tensor of the aligned SWCNT film. $\varepsilon_{0}$ is the vacuum permittivity, and $Q_{e}$ denotes the light power absorption density. The absorbed light power becomes heat, so $Q_{e}$ is the distributed heat source term in Equation (4). When a far infrared Gaussian beam (waist around $800 \mu \mathrm{m}$ ) is incident on the aligned SWCNT film ( $1 \mathrm{~mm}$ long, $5 \mu \mathrm{m}$ wide, and $2 \mu \mathrm{m}$ thick, as shown in Figure 1a), the light absorption distribution and the temperature rise distribution are simulated as the plots in Figure 1b,c. The frequency of the incident light is $1 \mathrm{THz}$ and the beam waist is $400 \mu \mathrm{m}$. The simulations are based on the finite element method and through the software COMSOL. After the temperature distribution over the SWCNT film is worked out, the photovoltage $(\Delta V)$ due to 
the PTE effect ( $\Delta V=\left(S_{p}-S_{n}\right) \Delta T$ ) is obtained. $S_{\mathrm{p}}$ and $S_{\mathrm{n}}$ denote the Seebeck coefficients of the p-doped and the n-doped regions, respectively. The details of simulation settings can be found in a previous study [18]. Since the real part of the parallel permittivity of the aligned SWCNT film $\left(\operatorname{Re}\left(\varepsilon_{\|}\right)\right)$is negative in the frequency range from 0 to $2.8 \mathrm{THz}$ [18], the film with a limited width (i.e., the length of the SWCNTs) can intrinsically support a localized surface plasmon (LSPR) mode. The LSPR frequency depends on the $W_{\text {film }}$. Although the LSPR of the aligned SWCNT film could also enhance the light absorption, the light coupling efficiency is low and the light field is not concentrated at the junction region. Thus, the responsivity enhancement and the polarization extinction ratio are 1 to 2 orders of magnitudes smaller than those induced by an optical antenna [18].

\section{Results}

In order to increase the responsivity and the polarization extinction ratio of the aligned SWCNT film based detector, it is natural to add an antenna to concentrate the incident light at the junction region of the SWCNT film, as shown in Figure 2a. The SWCNT film is $1 \mathrm{~mm}$ long, $5 \mu \mathrm{m}$ wide, and $2 \mu \mathrm{m}$ thick, as shown in Figure 1a. The antenna has a bowtie shape. The length $L_{\mathrm{a}}$ and the width $W_{\mathrm{a}}$ are both $150 \mu \mathrm{m}$. The feed point overlaps the junction region. There is an extension bar ( $20 \mu \mathrm{m}$ long and $2 \mu \mathrm{m}$ wide) at the tip of each wing. It is also $2 \mu \mathrm{m}$ away from the SWCNT film. Although an ordinary bowtie antenna can enhance the local $Q_{\mathrm{e}}$ at the junction by 240 times and thus enhance the responsivity by 23 times [18], the absolute absorptance of the junction region $\left(A_{\mathrm{j}}\right)$ is only $0.6 \%$. In order to further enhance $A_{\mathrm{j}}$ and the responsivity, we propose to integrate a bottom metal plane at a specific distance from the SWCNT film and the antenna, as shown in Figure 2b. As a result, the $Q_{\mathrm{e}}$ at the junction is further increased by 18 times (Figure $2 \mathrm{c}-\mathrm{e}$ ), and $\Delta T$ is further enhanced by 17.5 times (Figure $2 \mathrm{f}$ ). $A_{\mathrm{j}}$ is enhanced from $0.6 \%$ to $11.7 \%$, which is more than 3 orders of magnitude higher than that of the device without any antenna. The antenna dimensions $W_{a}$ and $L_{a}$ are adjusted from $150 \mu \mathrm{m}$ to $171 \mu \mathrm{m}$ after the bottom metal plane is integrated to keep the resonant frequency at $1 \mathrm{THz}$. When the thickness of the dielectric layer is as small as $13 \mu \mathrm{m}$, each wing of the bowtie antenna couples with its image due to the deep subwavelength thickness of the dielectric spacer and then forms a magnetic dipole, as exhibited in Figure 2c,e. At the magnetic dipole resonance, the incident light is efficiently coupled into the system, leading to an enhanced local field that significantly improves the responsivity of the carbon nanotube detector. The resonant frequency of the engineered bowtie antenna can be estimated by the antenna dimensions based on a patch antenna model [28]. The performance enhancement by the antenna engineering is attributed to the improved light coupling efficiency.

The local field intensity at the junction region can be derived from the coupled mode theory as [29]

$$
\frac{\left|E_{\mathrm{loc}}\right|^{2}}{\left|E_{0}\right|^{2}}=\frac{2 A_{\mathrm{c}} \lambda_{\text {res }}}{\pi} \frac{Q}{Q_{\mathrm{rad}}} \frac{Q}{V_{\text {eff }}}
$$

$E_{\text {loc }}$ is the amplitude of the local field, $E_{0}$ the amplitude of the incident light, $A_{\mathrm{c}}$ is the effective aperture, $\lambda_{\text {res }}$ the resonant wavelength, $V_{\text {eff }}$ the effective mode volume. $Q$ as the total quality factor is related to the radiation quality factor $\left(Q_{\text {rad }}\right)$ and the absorption quality factor $\left(Q_{\mathrm{abs}}\right)$ through the equation $Q^{-1}=Q_{\mathrm{rad}}{ }^{-1}+Q_{\mathrm{abs}}{ }^{-1}$. Based on the derivative of $\left|E_{\mathrm{loc}}\right|^{2} /\left|E_{0}\right|^{2}$ with respect to $Q_{\mathrm{rad}}$, it is revealed that $\left|E_{\mathrm{loc}}\right|^{2} /\left|E_{0}\right|^{2}$ reaches a maxima when $Q_{\mathrm{rad}}=Q_{\mathrm{abs}}$, which is called a critical coupling condition or an impedance matching condition. When the system reaches a critical coupling status, the light coupling efficiency becomes a maxima. $Q_{\text {abs }}$ is decided by the absorptance of the SWCNT film and the metal. In the absence of the bottom metal plane, the light mode is not very well confined (Figure 3a,b), and $Q_{\text {rad }}$ is much smaller than $Q_{\mathrm{abs}}$, so the system is far from a critical coupling status. In the presence of the bottom metal plane, the radiation from the antenna and that from the mirror image of the induced charges will constructively or destructively interfere with each other as the thickness of the dielectric layer varies (Figure 3c,d), so $Q_{\text {rad }}$ is tuned and the light coupling efficiency changes accordingly (Figure 3e). At a specific dielectric 
thickness, $Q_{\text {rad }}$ matches $Q_{\text {abs }}$ so the system reaches the critical coupling status and the light coupling efficiency is maximized. As revealed in Figure $3 b, d$, the local field induced by the optimized metal plane integrated antenna is several tens of times higher than that induced by the ordinary antenna.

(a)

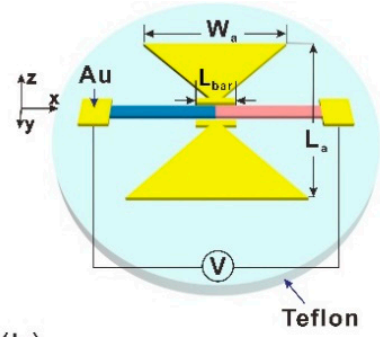

(b)

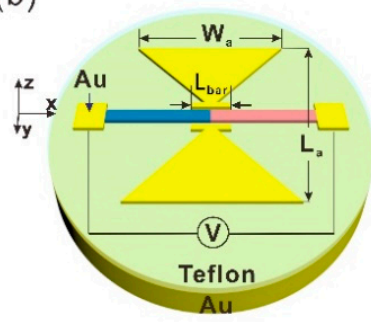

(c)

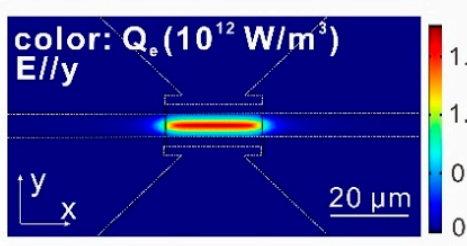

(d)

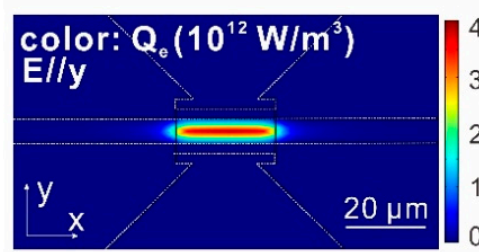

(e)
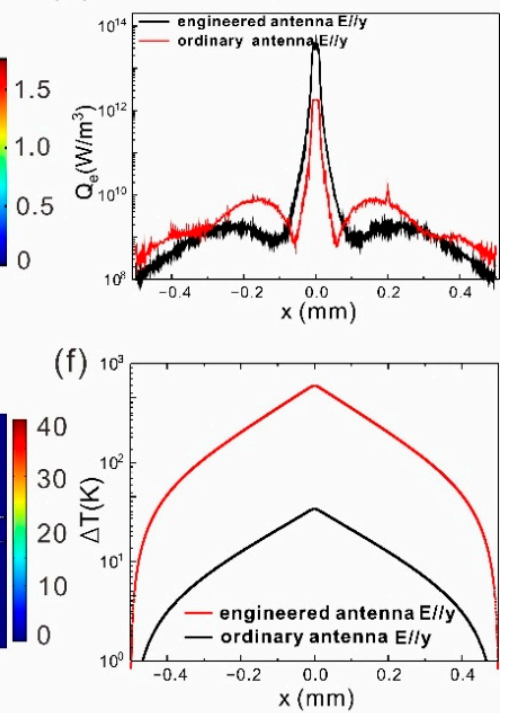

Figure 2. (a) Schematic of the ordinary antenna integrated aligned SWCNT film. $L_{\mathrm{a}}=W_{\mathrm{a}}=150 \mu \mathrm{m}, L_{\mathrm{bar}}=20 \mu \mathrm{m}$. (b) Schematic of the engineered antenna integrated aligned SWCNT film. A gold plane is added under the teflon layer. $L_{\mathrm{a}}=W_{\mathrm{a}}=171 \mu \mathrm{m}, L_{\mathrm{bar}}=20 \mu \mathrm{m}$. (c) Distribution of $Q_{\mathrm{e}}$ on the $x-y$ section at $z=1 \mu \mathrm{m}$, cutting through the center of the ordinary antenna integrated aligned SWCNT film. (d) Distribution of $Q_{\mathrm{e}}$ in the same way as (c) for the engineered antenna integrated aligned SWCNT film. (e) $Q_{\mathrm{e}}$ varying along the $x$-axis at $y=0 \mu \mathrm{m}$ and $z=1 \mu \mathrm{m}$ through the center of the SWCNT film for the ordinary antenna integrated device (red line) and the engineered antenna integrated device (black line). (f) $\Delta T$ varying along the $x$-axis at $y=0 \mu \mathrm{m}$ and $z=1 \mu \mathrm{m}$ through the center of the SWCNT film for the ordinary antenna integrated device (red line) and the engineered antenna integrated device (black line). In this figure, the incident light is polarized in the $y$-direction.

The length of the extension bar at each tip of the two wings of the bowtie antenna is an important parameter that has a big impact on the coupling efficiency. As shown in Figure $4 \mathrm{a}, A_{\mathrm{j}}$ varies non-monotonously with the length of the extension bar $\left(L_{\mathrm{bar}}\right)$. The highest junction region absorptance occurs at $L_{\mathrm{bar}}=40 \mu \mathrm{m}$. Correspondingly, the $\Delta T$ at the junction and the responsivity of the device also reaches the maxima at $L_{\mathrm{bar}}=40 \mu \mathrm{m}$ (Figure $4 \mathrm{~b}$, red line). This effect is attributed to the fine tuning of the antenna efficiency. According to the antenna theory [30], the antenna efficiency gets maximized when the radiation resistance $\left(R_{\mathrm{rad}}\right)$ equals to the load resistance $\left(R_{\text {load }}\right)$. Concerning the bowtie antenna without the bottom metal plane, $R_{\text {rad }}$ is around $73 \Omega$ [30]. $R_{\text {load }}$ is decided by the portion of the SWCNT film within the feed point region (defined by $L_{\text {bar }}$ and marked out by the purple frame in Figure $4 \mathrm{a})$. As $L_{\mathrm{bar}}$ increases, there are more and more SWCNTs bridging the feed point region in parallel, so $R_{\text {load }}$ decreases. $R_{\text {load }}=(1 / \sigma)\left(1 / L_{\text {bar }}\right)$ can be calculated through the conductivity of the SWCNT film $(\sigma=i \omega \varepsilon)$. $\omega$ is the angular frequency, $\varepsilon$ is the dielectric constant. Based on this expression, $R_{\text {load }}$ is calculated to be $158 \Omega$ at $L_{\text {bar }}=20 \mu \mathrm{m}$, and $78 \Omega$ at $L_{\text {bar }}=60 \mu \mathrm{m}$. $R_{\text {load }}$ is close to $R_{\text {rad }}$ at $L_{\text {bar }}=60 \mu \mathrm{m}$, so the antenna efficiency and thus the junction region absorptance reach a maxima in this condition (Figure 4a black line). When the antenna is integrated with a metal plane, the length of the bowtie antenna $\left(L_{\mathrm{a}}\right)$ needs to be enlarged to maintain the resonant wavelength. Since $R_{\text {load }}$ increases with $L_{\mathrm{a}}$, the situation $R_{\text {rad }}=R_{\text {load }}$ occurs at a smaller $L_{\text {bar }}$ for the metal plane integrated antenna. As confirmed by the red line in Figure $4 b, c$, the optimized $A_{\mathrm{j}}$ appears around $L_{\mathrm{bar}}=40 \mu \mathrm{m}$. In this case, the engineered antenna with $L_{\mathrm{bar}}=40 \mu \mathrm{m}$ 
enhances $A_{\mathrm{j}}$ by 21.3 times and enhances $\Delta T\left(R_{\mathrm{V}}\right)$ further by 19.9 times in comparison with the case of the ordinary bowtie antenna integrated device.

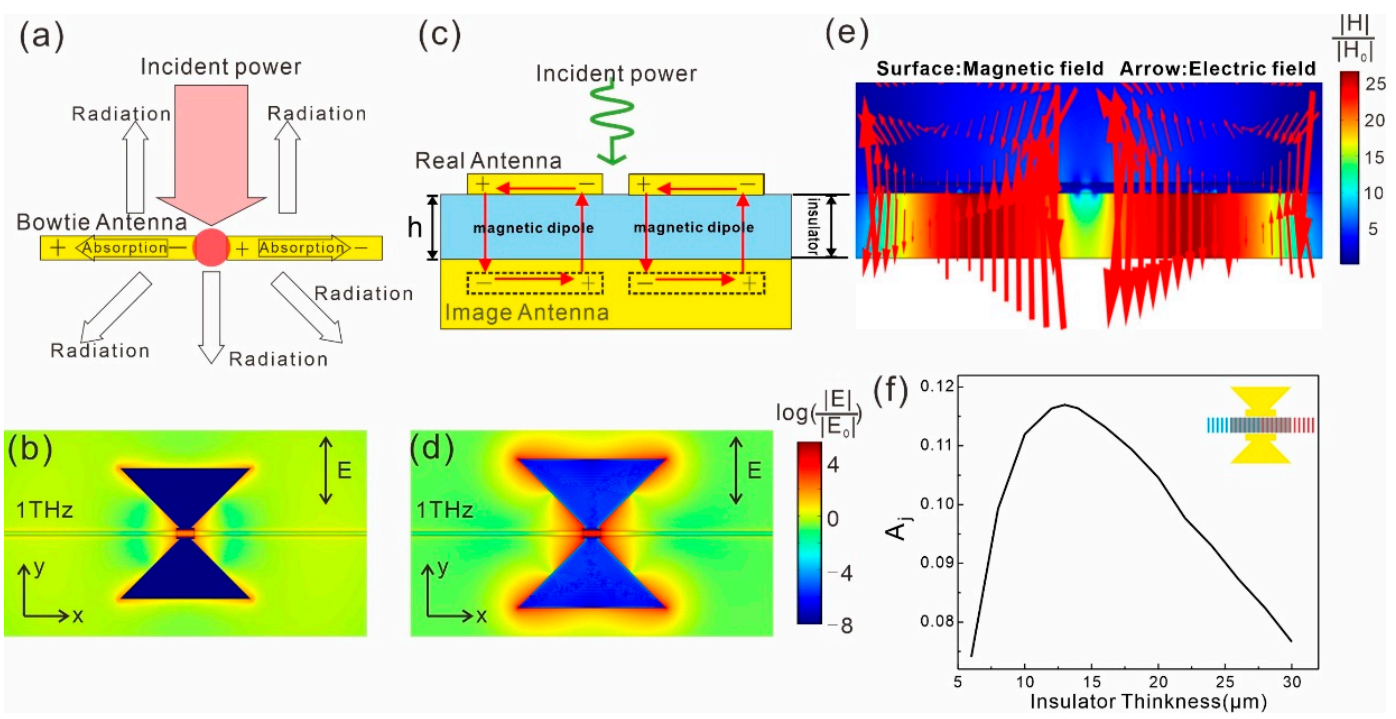

Figure 3. (a) Schematic diagram of modeling a bowtie antenna as a resonator using coupled mode theory. (b) Electric field enhancement distributions $\left(\log \left(|E| /\left|E_{0}\right|\right)\right)$ on the $x-y$ cross section at the center of the SWCNT film $(z=1 \mu \mathrm{m})$ integrated with the bowtie antenna at $1 \mathrm{THz}$ for $y$-polarization. (c) Schematic diagram of the engineered antenna integrated SWCNT film. Each wing couples with its image and induces a magnetic dipole. (d) Same as (b) for the SWCNT film integrated with an engineered antenna (with bottom metal plane). (e) Electromagnetic field distribution of the engineered antenna integrated SWCNT film on a $y-z$ cross section at $x=0$. (f) $A_{\mathrm{j}}$ as a function of the thickness of the insulator spacer in the engineered antenna integrated SWCNT film for $y$-polarization at $1 \mathrm{THz}$. The junction area is defined as a $5 \mu \mathrm{m} \times 100 \mu \mathrm{m} \times 2 \mu \mathrm{m}$ box at the center of the SWCNT film, as shown by the gray shade in the inset. $E_{0}$ in $(\mathbf{b}, \mathbf{d})$ denotes the electric field of the incident Gaussian beam at the junction.
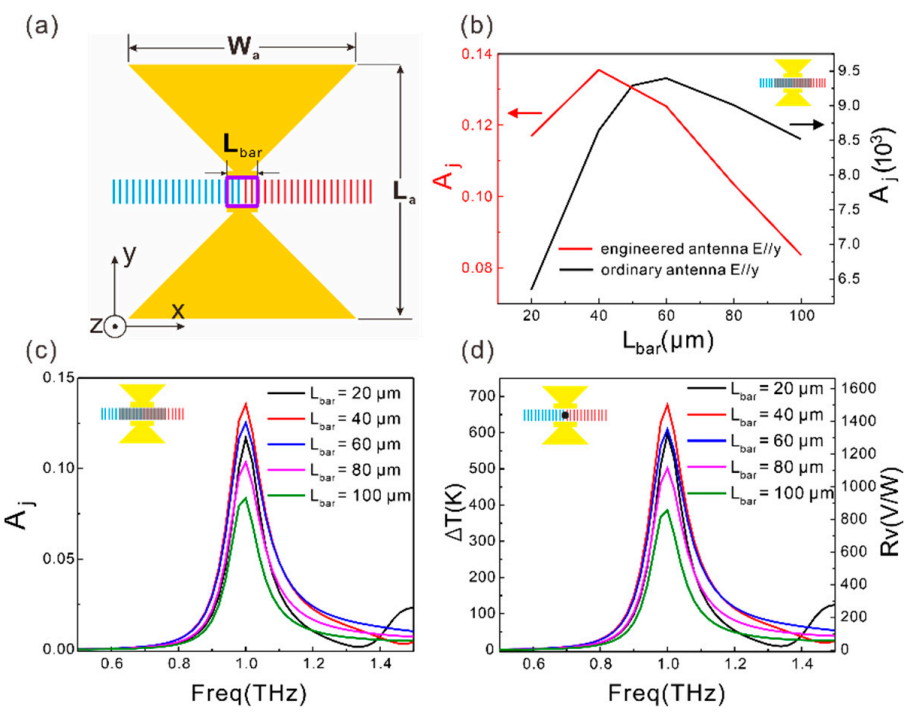

Figure 4. (a) Schematic diagram of the bowtie antennas integrated aligned SWCNT film. The purple region represents the part of the SWCNT film between the two extension bars. $R_{\text {load }}$ is decided by the SWCNTs within this region. (b) $A_{\mathrm{j}}$ as a function of $L_{\mathrm{bar}}$ for $y$-polarized incident light at $1 \mathrm{THz}$. Black line: ordinary antenna integrated aligned SWCNT film; Red line: engineered antenna integrated aligned SWCNT film. (c) Spectra of $A_{\mathrm{j}}$ at different $L_{\mathrm{bar}}$ for the engineered antenna integrated SWCNT film for $y$-polarization. (d) Spectra of the junction temperature increase $(\Delta T)$ and the responsivity at different $L_{\mathrm{bar}}$ for the engineered antenna integrated SWCNT film for $y$-polarized light. when $L_{\text {bar }}=20,40,60,80$ or $100 \mu \mathrm{m}, L_{\mathrm{a}}=W_{\mathrm{a}}=171,154,133,115$ or $100 \mu \mathrm{m}$, respectively. 
The spectra of $A_{\mathrm{j}}, \Delta T$, and $R_{\mathrm{V}}$ for the SWCNT infrared detector integrated with the ordinary antenna and that with the engineered antenna are presented in Figure $5 \mathrm{a}, \mathrm{b}$. As confirmed by the spectra in Figure $5 a, b$, the engineered antenna enhances the peak absorptance and $\Delta T\left(R_{\mathrm{V}}\right)$ significantly at the resonant frequency for $y$-polarized incident light. The peak $R_{\mathrm{V}}$ reaches $1500 \mathrm{~V} / \mathrm{W}$, which is 19.9 times higher than that achieved by an ordinary antenna. For the $x$-polariztion, the engineered antenna induces an $R_{\mathrm{V}}$ of $0.045 \mathrm{~V} / \mathrm{W}$ at $1 \mathrm{THz}$ and the ordinary antenna induces an $R_{\mathrm{V}}$ of $0.043 \mathrm{~V} / \mathrm{W}$. Therefore, the engineered antenna enhances the PER by 19.1 times to 33,440. By altering the dimension of the antenna, the peak responsivity can be set at different frequencies. As shown in Figure $5 \mathrm{c}$, as the antenna dimension rising from $95 \mu \mathrm{m}$ to $379 \mu \mathrm{m}$, the peak responsivity shifts from $1.5 \mathrm{THz}$ to $0.5 \mathrm{THz}$ in the frequency domain. Concerning the antenna as large as $379 \mu \mathrm{m}$, it also supports higher order modes in this frequency range, as shown by the pink line in Figure $5 \mathrm{c}$. The peak $R_{\mathrm{V}}$, peak PER, and the enhancement times of these two quantities compared to that of the ordinary antenna integrated devices are presented in Table 1.

(a)

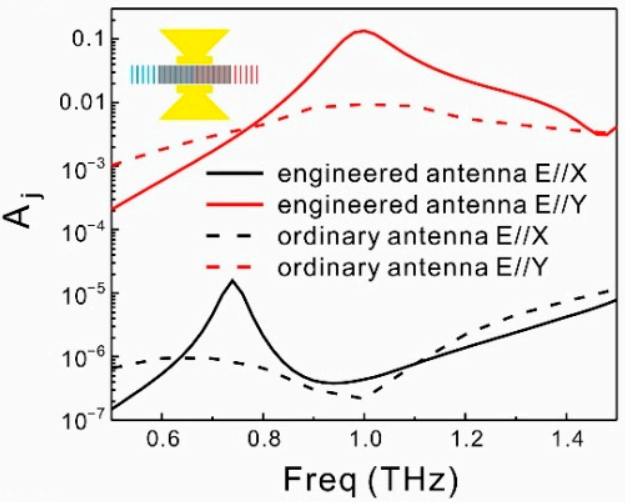

(c)

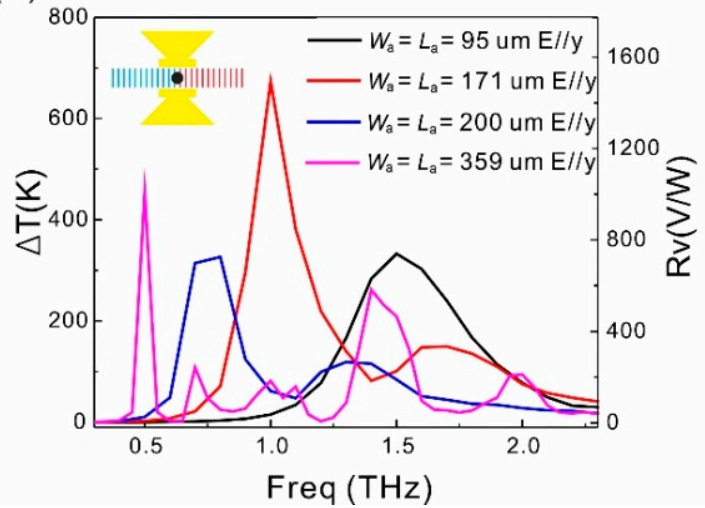

(b)

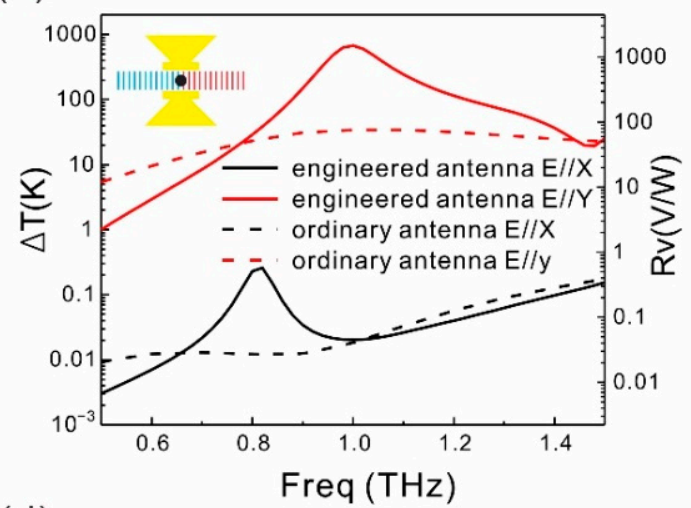

(d)

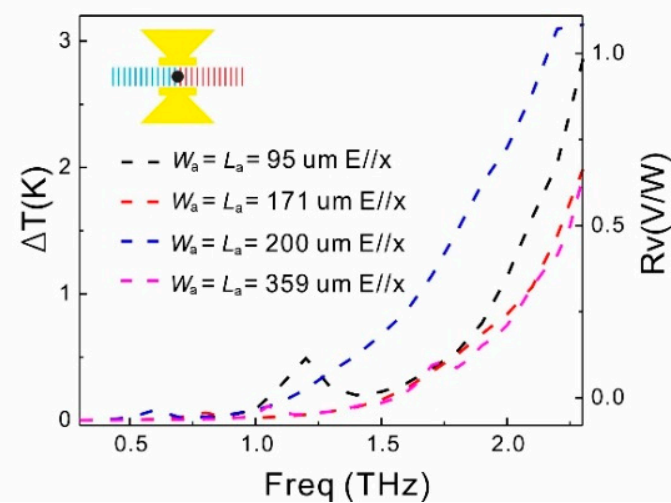

Figure 5. (a) Spectra of $A_{\mathrm{j}}$ of the ordinary antenna integrated SWCNT film and that of the engineered antenna integrated SWCNT film for $x$ - and $y$-polarized incident light at $1 \mathrm{THz}$. (b) Spectra of $\Delta T$ and $R_{\mathrm{V}}$ of the ordinary antenna integrated SWCNT film and the engineered antenna integrated SWCNT film for $x$ - and $y$-polarized incident light at $1 \mathrm{THz}$. (c,d) Spectra of $\Delta T$ and $R_{\mathrm{V}}$ of the engineered antenna integrated SWCNT film for four different antenna sizes $\left(L_{\mathrm{a}}=W_{\mathrm{a}}=95 \mu \mathrm{m}, 150 \mu \mathrm{m}\right.$, $200 \mu \mathrm{m}, 379 \mu \mathrm{m})$ excited by $y$ - and $x$-polarized light, respectively. 
Table 1. Peak Rv, peak PER, and enhancement times of Rv and PER compared to that of the ordinary antenna integrated devices.

\begin{tabular}{ccccc}
\hline & $\begin{array}{c}\text { Insulator } \\
\text { Thinkness }\end{array}$ & Peak Rv & Peak PER & $\begin{array}{c}\text { Enhancement of } \\
\text { Peak Rv }\end{array}$ \\
\hline $\begin{array}{c}\text { Engineered antenna } \\
L_{\mathrm{a}}=W_{\mathrm{a}}=95 \mu \mathrm{m} \\
\text { Engineered antenna } \\
L_{\mathrm{a}}=W_{\mathrm{a}}=171 \mu \mathrm{m}\end{array}$ & $17 \mu \mathrm{m}$ & $735.5 \mathrm{~V} / \mathrm{W} @ 1.5 \mathrm{THz}$ & $1481.1 @ 1.5 \mathrm{THz}$ & $8.1 @ 1.5 \mathrm{THz}$ \\
$\begin{array}{c}\text { Engineered antenna } \\
L_{\mathrm{a}}=W_{\mathrm{a}}=200 \mu \mathrm{m}\end{array}$ & $13 \mu \mathrm{m}$ & $1500 \mathrm{~V} / \mathrm{W} @ 1 \mathrm{THz}$ & $33400 @ 1 \mathrm{THz}$ & $19.9 @ 1 \mathrm{THz}$ \\
$\begin{array}{c}\text { Engineered antenna } \\
L_{\mathrm{a}}=W_{\mathrm{a}}=379 \mu \mathrm{m}\end{array}$ & $32 \mu \mathrm{m}$ & $693.7 \mathrm{~V} / \mathrm{W} @ 0.7 \mathrm{THz} \mathrm{THz}$ & $14732.8 @ 0.7 \mathrm{THz}$ & $9.3 @ 0.7 \mathrm{THz}$ \\
\hline
\end{tabular}

\section{Conclusions}

In conclusion, we numerically demonstrated that light coupling engineering of an optical antenna integrated aligned SWCNT film based far infrared detector can further significantly enhance the performance. By integrating a bottom metal plane at a specific distance from the antenna and tuning the length of the extension bar at the tip of each wing of the antenna, the peak responsivity is further enhanced by 8.1 to 19.9 times over the frequency range from $0.5 \mathrm{THz}$ to $1.5 \mathrm{THz}$, and the polarization extinction ratio is enhanced by 2.7 to 22.3 times. The highest peak responsivity in this range reaches $1500 \mathrm{~V} / \mathrm{W}$ at $1 \mathrm{THz}, 19.9$ times higher than that induced by the ordinary antenna. The peak polarization extinction ratio is higher than 1481 over the range from 0.5 to $1.5 \mathrm{THz}$ and reaches an extremely high value of $3.04 \times 10^{5}$ at $0.5 \mathrm{THz}$. The mechanism of the enhancement is attributed to critical coupling and impedance matching, and this principle can be applied to a variety of systems to improve the light-matter interaction in subwavelength scales.

Author Contributions: Conceptualization, J.Z. and X.C.; methodology, X.R.; software, X.R., Z.C. and Z.J.; validation, X.R., J.Z. and B.C.; formal analysis, X.R. and Z.C.; investigation, X.R.; resources, J.Z.; data curation, X.R. and Z.J.; writing—original draft preparation, X.R. and Z.J.; writing-review and editing, J.Z. and X.C.; visualization, X.R.; supervision, J.Z. and X.C.; project administration, J.Z. and X.C.; funding acquisition, J.Z. and X.C. All authors have read and agreed to the published version of the manuscript.

Funding: This research was funded by the National Key Research and Development Program of China (2017YFA0205800, 2018YFA0306200), the National Natural Science Foundation of China (61975223, 61874126, 91850208, 61991442, 61521005), the Hundred Talents Program of the Chinese Academy of Sciences (No. 20181214), the Key Deployment Projects of the Chinese Academy of Sciences (ZDRW-XH-2021-7-1), the Fund of Shanghai Science and Technology Foundation (18JC1420401), the Shanghai Municipal Science and Technology Major Project (Grant No. 2019SHZDZX01), the Shenzhen Science and Technology Program (Grant No. KQTD20190929173954826).

Institutional Review Board Statement: Not applicable.

Informed Consent Statement: Not applicable.

Acknowledgments: The authors acknowledge the support by the National Key Research and Development Program of China (2017YFA0205800, 2018YFA0306200), the National Natural Science Foundation of China (61975223, 61874126, 91850208, 61991442, 61521005), the Hundred Talents Program of the Chinese Academy of Sciences (No. 20181214), the Key Deployment Projects of the Chinese Academy of Sciences (ZDRW-XH-2021-7-1), the Fund of Shanghai Science and Technology Foundation (18JC1420401), the Shanghai Municipal Science and Technology Major Project (Grant No. 2019SHZDZX01), the Shanghai Tech University Quantum Device Lab; Shenzhen Science and Technology Program (Grant No. KQTD20190929173954826).

Conflicts of Interest: The authors declare no conflict of interest. 


\section{References}

1. Tonouchi, M. Cutting-edge terahertz technology. Nat. Photonics 2007, 1, 97-105. [CrossRef]

2. Lee, M.; Wanke, M.C. Searching for a Solid-State Terahertz Technology. Science 2007, 316, 64-65. [CrossRef] [PubMed]

3. Mittleman, D. Frontiers in terahertz sources and plasmonics. Nat. Photonics 2013, 7, 666-669. [CrossRef]

4. He, X.; Léonard, F.; Kono, J. Uncooled Carbon Nanotube Photodetectors. Adv. Opt. Mater. 2015, 3, 989-1011. [CrossRef]

5. Suen, J.Y.; Fan, K.; Montoya, J.; Bingham, C.; Stenger, V.; Sriram, S.; Padilla, W.J. Multifunctional metamaterial pyroelectric infrared detectors. Optica 2017, 4, 276-279. [CrossRef]

6. Kulkarni, E.S.; Heussler, S.P.; Stier, A.V.; Martin-Fernandez, I.; Andersen, H.; Toh, C.T.; Özyilmaz, B. Exploiting the IR Transparency of Graphene for Fast Pyroelectric Infrared Detection. Adv. Opt. Mater. 2015, 3, 34-38. [CrossRef]

7. Li, L.; Zhao, X.; Li, X.; Ren, B.; Xu, Q.; Liang, Z.; Di, W.; Yang, L.; Luo, H.; Shao, X.; et al. Scale Effects of Low-Dimensional Relaxor Ferroelectric Single Crystals and Their Application in Novel Pyroelectric Infrared Detectors. Adv. Mater. 2014, 26, 2580-2585. [CrossRef]

8. St-Antoine, B.C.; Menard, D.; Martel, R. Single-Walled Carbon Nanotube Thermopile for Broadband Light Detection. Nano Lett. 2011, 11, 609-613. [CrossRef]

9. Krause, B.; Bezugly, V.; Khavrus, V.; Ye, L.; Cuniberti, G.; Pötschke, P. Boron Doping of SWCNTs as a Way to Enhance the Thermoelectric Properties of Melt-Mixed Polypropylene/SWCNT Composites. Energies 2020, 13, 394. [CrossRef]

10. Corletto, A.; Shapter, J.G. Discontinuous Dewetting, Template-Guided Self-Assembly, and Liquid Bridge-Transfer Printing of High-Resolution Single-Walled Carbon Nanotube Lines for Next-Generation Electrodes and Interconnects. ACS Appl. Nano Mater. 2020, 3, 8148-8160. [CrossRef]

11. Cai, B.; Yin, H.; Huo, T.T.; Ma, J.; Di, Z.; Li, M.; Hu, N.; Yang, Z.; Zhang, Y.; Su, Y. Semiconducting single-walled carbon nanotube/graphene van der Waals junctions for highly sensitive all-carbon hybrid humidity sensors. J. Mater. Chem. C 2020, 8 , 3386-3394. [CrossRef]

12. Hadjichristov, G.B.; Exner, G.K.; Marinov, Y.G.; Vlakhov, T.E. Photo-electrical response of nanocomposites from single-walled carbon nanotubes incorporated in tris(keto-hydrozone) discotic mesogen. J. Phys. Conf. Ser. 2021, 1762, 012011. [CrossRef]

13. He, X.; Fujimura, N.; Lloyd, J.M.; Erickson, K.J.; Talin, A.A.; Zhang, Q.; Gao, W.; Jiang, Q.; Kawano, Y.; Hauge, R.H.; et al. Carbon Nanotube Terahertz Detector. Nano Lett. 2014, 14, 3953-3958. [CrossRef]

14. He, X.; Wang, X.; Nanot, S.; Cong, K.; Jiang, Q.; Kane, A.A.; Goldsmith, J.E.M.; Hauge, R.H.; Léonard, F.; Kono, J. Photothermoelectric p-n Junction Photodetector with Intrinsic Broadband Polarimetry Based on Macroscopic Carbon Nanotube Films. ACS Nano 2013, 7, 7271-7277. [CrossRef]

15. Agio, M.; Alù, A. Optical Antennas; Cambridge University Press: Cambridge, UK, 2013.

16. Novotny, L.; Van Hulst, N. Antennas for light. Nat. Photonics 2011, 5, 83-90. [CrossRef]

17. Giannini, V.; Fernandez-Dominguez, A.I.; Heck, S.C.; Maier, S. Plasmonic Nanoantennas: Fundamentals and Their Use in Controlling the Radiative Properties of Nanoemitters. Chem. Rev. 2011, 111, 3888-3912. [CrossRef]

18. Chen, B.; Ji, Z.; Zhou, J.; Yu, Y.; Dai, X.; Lan, M.; Bu, Y.; Zhu, T.; Li, Z.; Hao, J.; et al. Highly polarization-sensitive far infrared detector based on an optical antenna integrated aligned carbon nanotube film. Nanoscale 2020, 12, 11808-11817. [CrossRef] [PubMed]

19. Sihvola, A.H. Electromagnetic Mixing Formulas and Applications; Institution of Electrical Engineers: London, UK, 1999.

20. Han, J.; Zhu, Z.; Liao, Y.; Wang, Z.; Yu, L.; Zhang, W.; Sun, L.; Wang, T. Optical conductivity of single walled nanotube films in the Terahertz region. JETP Lett. 2003, 78, 436-439. [CrossRef]

21. Wang, M.Y.; Li, H.; Zhang, M.; Dong, Y.; Zhong, C. Terahertz Wave Characteristic of Single Walled Carbon Nanotubes Using Propagation Matrix Method. Frequenz 2015, 69, 419-425. [CrossRef]

22. Pint, C.L.; Xu, Y.-Q.; Pasquali, M.; Hauge, R.H. Formation of Highly Dense Aligned Ribbons and Transparent Films of SingleWalled Carbon Nanotubes Directly from Carpets. ACS Nano 2008, 2, 1871-1878. [CrossRef] [PubMed]

23. Gao, Y.; Marconnet, A.M.; Xiang, R.; Maruyama, S.; Goodson, K.E. Heat Capacity, Thermal Conductivity, and Interface Resistance Extraction for Single-Walled Carbon Nanotube Films Using Frequency-Domain Thermoreflectance. IEEE Trans. Compon. Packag. Manuf. Technol. 2013, 3, 1524-1532. [CrossRef]

24. Jeon, T.-I.; Kim, K.-J.; Kang, C.; Oh, S.-J.; Son, J.-H.; An, K.H.; Bae, D.J.; Lee, Y.H. Terahertz conductivity of anisotropic single walled carbon nanotube films. Appl. Phys. Lett. 2002, 80, 3403-3405. [CrossRef]

25. Roberts, J.A.; Yu, S.-J.; Ho, P.-H.; Schoeche, S.; Falk, A.L.; Fan, J.A. Tunable Hyperbolic Metamaterials Based on Self-Assembled Carbon Nanotubes. Nano Lett. 2019, 19, 3131-3137. [CrossRef]

26. Xia, J.-Y.; Dong, G.-D.; Tian, B.-Y.; Yan, Q.-P.; Han, J.; Qiu, S.; Li, Q.; Liang, X.-L.; Peng, L.-M. Contact Resistance Effects in Carbon Nanotube Thin Film Transistors. Acta Phys. Chim. Sin. 2016, 32, 1029-1035. [CrossRef]

27. Han, K.N.; Li, C.A.; Bui, M.-P.N.; Seong, G.H. Patterning of Single-Walled Carbon Nanotube Films on Flexible, Transparent Plastic Substrates. Langmuir 2010, 26, 598-602. [CrossRef] [PubMed]

28. Nahar, T.; Sharma, O.P. A Modified Multiband Bow Tie Antenna Array used for L band Application. Int. J. Eng. Res. Technol. 2014, 3, 81-85.

29. Seok, T.J.; Jamshidi, A.; Kim, M.; Dhuey, S.; Lakhani, A.; Choo, H.; Schuck, P.J.; Cabrini, S.; Schwartzberg, A.; Bokor, J.; et al. Radiation Engineering of Optical Antennas for Maximum Field Enhancement. Nano Lett. 2011, 11, 2606-2610. [CrossRef] [PubMed]

30. Balanis, C.A. Antenna Theory: Analysis and Design; Wiley: Hoboken, NJ, USA, 2012. 